\title{
Estandarización de un medio de cultivo a partir de fuentes agroindustriales para la multiplicación de Azospirillum brasilense ${ }^{1}$
}

\author{
\begin{tabular}{l|l|l} 
Diego Rivera $^{2}$ & Melissa Obando & Ruth Bonilla Buitrago
\end{tabular}
}

Recibido:

Junio 20 de 2012

Aceptado:

Noviembre 30 de 2012

${ }^{1}$ El artículo fue desarrollado con base a una investigación, financiada por la Corporación

Colombiana de Investigación

Agropecuaria-Corpoica, en

el marco del proyecto con

alianza con el Ministerio

de Agricultura y Desarrollo Rural-MADR "Producción de fertilizantes biológicos a partir

del género Azospirillum sp. para mejorar la productividad y sostenibilidad de gramíneas en

suelos del valle del cesar".

${ }^{2}$ M.Sc Ciencias Farmacéuticas

Universidad Nacional de

Colombia-Facultad de Ciencias,

Corporación Colombiana de

Investigación AgropecuariaCorpoica, Centro de

Biotecnología y Bioindustria-

Correo electrónico: ing.diegorivera@hotmail.com

${ }^{3} \mathrm{MSc}$ Ciencias AgrariasFisiología de Cultivos

Universidad Nacional de

Colombia-Facultad de

Agronomía, Corporación

Colombiana de Investigación

Agropecuaria-Corpoica, Centro

de Biotecnología y

Bioindustria-CBB

Correo electrónico:

dmobandoc@unal.edu.co

${ }^{4} \mathrm{Ph}$. D. Ciencias agrícolas. Líder

Laboratorio de Microbiología

de Suelos, Corporación

Colombiana de Investigación

Agropecuaria-Corpoica, Centro

de Biotecnología y

Bioindustria-CBB

Correo electrónico:

\section{Resumen}

Se realizó una etapa exploratoria aplicando el diseño Plackett-Burman para cuatro fuentes de carbono, dos fuentes de nitrógeno, $\mathrm{K}_{2} \mathrm{HPO}_{4}$ y $\mathrm{MgSO}_{4}$ y una solución de micronutrientes con el fin de seleccionar los factores nutricionales más influyentes sobre el crecimiento del microorganismo. Posteriormente, se llevó a cabo un proceso de optimización aplicando el diseño Box-Behnken de los tres factores más influyentes; los resultados indicaron que la producción de biomasa (UFC/mL) fue afectada significativamente por condiciones tanto nutricionales como físicas del medio de cultivo; el máximo rendimiento obtenido fue de $1 \times 10^{9} \mathrm{UFC} / \mathrm{mL}$, el cual se logró bajo las siguientes condiciones: $3 \mathrm{~g} / \mathrm{L}$ sacarosa, 6,75 $\mathrm{mL} / \mathrm{L}$ melaza, 0,77 $\mathrm{mL} / \mathrm{L}$ glicerol, 14,42 mL/L hidrolizado de soya, 0,848 g/L hidrolizado de levadura, 0,25 g/L $\mathrm{K}_{2} \mathrm{HPO}_{4}, 3 / 5$ aireación y agitación a $120 \mathrm{rpm}$ con un tiempo de fermentación de 26 horas.

Palabras clave: Azospirillum brasilense, diseño Plackett-Burman, diseño Box-Behnken, superficie de respuesta.

\section{Abstract}

The Plackett-Burman statistical design was initially applied for Screening the most important nutritional variables (four carbon sources, two nitrogen sources, $\mathrm{K}_{2} \mathrm{HPO}_{4}$ y $\mathrm{MgSO}_{4}$ and a trace elements solution) affecting microorganism biomass production. Subsequently, a optimising the process by applied a BoxBehnken design for the most important factors tree. The results showed that biomass (UFC/mL) production was strongly affected by nutritional and physico conditions of culture medium. The highest yield was obtained in the following conditions: $3 \mathrm{~g} / \mathrm{L}$ sucrose, $6,75 \mathrm{~mL} / \mathrm{L}$ molasses, $0,77 \mathrm{~mL} / \mathrm{L}$ glycerol, 14,42 $\mathrm{mL} / \mathrm{L}$ soybean hydrolyzate, $0,848 \mathrm{~g} / \mathrm{L}$ yeast hidrolyzate, $0,25 \mathrm{~g} / \mathrm{L} \mathrm{K}_{2} \mathrm{HPO}_{4}, 3 / 5$ 
aeration and shaking at $120 \mathrm{rpm} 26$ hours total fermentation time during which $1 \times 10^{9} \mathrm{UFC} / \mathrm{mL}$ were obtained.

Keywords: Azospirillum brasilense, Plackett-Burman Design, Box-Behnken Design, Response surface.

\section{Introducción}

E 1 Cesar es uno de los principales departamentos ganaderos de la Costa Caribe y la mayor parte de su hato está dedicado a la producción conjunta de leche y de carne. Y es donde los cultivos de pasto juegan un papel importante en la alimentación y nutrición del ganado (Gamarra, 2005). Debido a esto, para que existan buenos rendimientos en cuanto a calidad nutricional y productividad del forraje se debe tener en cuenta el estado del suelo; por lo que actualmente este presenta un deterioro físico, químico y biológico afectando severamente su capacidad productiva a mediano y largo plazo, comprometiendo de esta manera la viabilidad económica de los sistemas ganaderos ocasionados por el uso desmedido de fertilizantes de síntesis química (Adesemoye et al., 2008; Cárdenas et al., 2010)

Por lo tanto, una alternativa viable que contribuye favorablemente con este propósito es el uso de los biofertilizantes (Dobbelaere et al., 2003; Baldani et al., 2008) los cuales recuperan la fertilidad y productividad del suelo y permiten darle a las plantas los nutrimentos necesarios para su crecimiento contribuyendo en este sentido a mejorar la calidad de los cultivos para su producción agrícola (Adesemoye et al., 2008).

De esta manera, es de gran importancia la multiplicación de Azospirillum spp. para mejorar la calidad del suelo y productividad de los cultivos (Díaz-Zorita y Fernández-
Canigia, 2009; Bashan et al., 2011), por lo que la aplicación de diseños estadísticos (Niladevi et al., 2009) secuenciales para la generación de un medio de cultivo económico se convierte en un factor determinante a la hora de producir un inoculante bacteriano, debido a los elevados costos de los nutrientes grado reactivo que son utilizados. Es por lo anterior que se requiere un proceso de desarrollo tecnológico que incluya la estandarización de las condiciones nutricionales, manejando los requerimientos fisiológicos mediante herramientas estadísticas que permitan obtener una aproximación de los factores que intervienen en la viabilidad celular (Niladevi et al., 2009).

De acuerdo a lo anterior, el objetivo de este estudio fue estandarizar un medio de cultivo alternativo para la multiplicación de Azospirillum brasilense C16, evaluando sustratos de bajo costo sobre el crecimiento máximo del microorganismo a escala de laboratorio.

\section{Materiales y métodos}

\section{Microorganismo y medio de cultivo}

La cepa C16 (Azospirillum brasilense) fue suministrada por el Banco de cepas del Laboratorio de Microbiología de Suelos del Centro de Biotecnología y Bioindustria (CBBCorpoica C.I Tibaitatá). El microorganismo fue mantenido en crioconservación a $-20^{\circ} \mathrm{C}$ y se reactivó en el medio de cultivo Dygs $\left(\mathrm{K}_{2} \mathrm{HPO}_{4} * 3 \mathrm{H}_{2} \mathrm{O}: 0,5 \mathrm{~g} / \mathrm{L}, \mathrm{MgSO}_{4} * 7 \mathrm{H}_{2} \mathrm{O}: 0,5\right.$ 
Estandarización de un medio de cultivo a partir de fuentes agroindustriales para la multiplicación de

g/L, glucosa: $2 \mathrm{~g} / \mathrm{L}$, peptona universal: $1,5 \mathrm{~g} / \mathrm{L}$, extracto de levadura: $2 \mathrm{~g} / \mathrm{L}$, ácido glutámico: $1,5 \mathrm{~g} / \mathrm{L}$, ácido málico: $2 \mathrm{~g} / \mathrm{L}$ ajustado a un $\mathrm{pH}$ de 7,0) (Rodríguez et al., 1986).

Estandarización del inóculo y determinación de la viabilidad celular

Se realizó una suspensión celular al 10\% en solución salina $(\mathrm{NaCl}$ 0,85\%) de la cepa C16 crecida a una temperatura de $30 \pm 2^{\circ} \mathrm{C}$ a 120 rpm durante 30 h, ajustada al tubo № 5 de la Escala de Mc Farland. La viabilidad celular se determinó mediante recuento en placa por la técnica de microgota (Doyle et al., 2000) sobre agar batata (Döbereiner et al., 1995), realizando diluciones seriadas en base diez desde $10^{-2}$ hasta $10^{-8}$. El tiempo de incubación fue de $48 \mathrm{~h}$ a $30 \pm 2^{\circ} \mathrm{C}$.

\section{Caracterización de los sustratos empleados}

Se utilizaron seis sustratos: sacarosa, melaza, glutamato, glicerol, soya y extracto de levadura. Se determinó el porcentaje de carbono orgánico por el método de WalkleyBlack modificado y nitrógeno total por el método Kjeldahl.

Cinética de crecimiento en el medio de cultivo de referencia y alternativo

Se realizó una suspensión celular al $10 \%(\mathrm{v} / \mathrm{v})$ de la bacteria en solución salina $(\mathrm{NaCl} 0,85 \%)$, la cual fue ajustada con el tubo № 5 de la Escala de Mc Farland. Ésta se llevó a un erlenmeyer de 2000 $\mathrm{mL}$, tomando una relación de aireación $(1 / 5)$ a $120 \mathrm{rpm}$ y una temperatura de $30 \pm 2{ }^{\circ} \mathrm{C}$; el ensayo fue realizado por triplicado tomando muestras cada 3 horas durante 48 horas.

\section{Análisis estadístico y de costos}

Se aplicó una etapa preliminar exploratoria -screening- usando el diseño Plackett-Burman (Plackett y Burman, 1946) y una etapa de optimización mediante la generación de una superficie de respuesta, con la aplicación del diseño Box-Behnken; el cual permite seleccionar los factores más influyentes (Box y Behnken, 1960). Se utilizó el software estadístico Statgraphics Plus (versión 5.1).

Se implementaron 14 tratamientos por triplicado, utilizando erlenmeyers de 125 $\mathrm{mL}$. Se evaluaron 9 factores nutricionales: cuatro fuentes de carbono (sacarosa, melaza, glutamato y glicerol), dos fuentes de nitrógeno (hidrolizado de soya e hidrolizado de extracto de levadura), dos sales $\left(\mathrm{K}_{2} \mathrm{HPO}_{4}\right.$ y $\left.\mathrm{MgSO}_{4}\right)$, solución de microelementos y dos factores físicos: aireación y agitación (Tabla 1). Cada factor se evaluó a dos niveles, un nivel alto denotado por $(+)$ y un nivel bajo designado por (-).

Tabla 1. Factores analizados con un diseño PlackettBurman, codificación de los factores y niveles manejados

\begin{tabular}{|lccc|}
\hline FACTOR (g/L) & SíMBOLO & \multicolumn{2}{c|}{ NIVELES DEL FACTOR } \\
& & Nivel bajo (-1) & Nivel alto (+1) \\
\hline $\mathrm{K}_{2} \mathrm{HPO}_{4}$ & $\mathrm{~S}_{1}$ & 0,25 & 0,75 \\
$\mathrm{MgSO}_{4}$ & $\mathrm{~S}_{2}$ & 0,25 & 0,75 \\
Sacarosa & $\mathrm{C}_{1}$ & 1 & 3 \\
Melaza & $\mathrm{C}_{2}$ & 0,675 & 2,025 \\
Glutamato & $\mathrm{C}_{3}$ & 0,74625 & 2,23875 \\
Glicerol & $\mathrm{C}_{4}$ & 0,98 & 2,94 \\
Hidrolizado de soya & $\mathrm{N}_{1}$ & 0,721 & 2,163 \\
Hidrolizado de extracto de & $\mathrm{N}_{2}$ & 0,848 & 2,544 \\
levadura & $\mathrm{I}$ & $1 / 5$ & $2 / 5$ \\
Aireación & $\mathrm{J}$ & $60 \mathrm{rpm}$ & $180 \mathrm{rpm}$ \\
Agitación & $\mathrm{K}$ & $0,5 \mathrm{ml} / \mathrm{L}$ & $1,5 \mathrm{ml} / \mathrm{L}$ \\
Microelementos & & & \\
\hline
\end{tabular}

\section{Resultados y discusión}

\section{Caracterización de los sustratos empleados}

Se obtuvieron los valores de concentración de carbono y nitrógeno total de las seis fuentes nutricionales utilizadas, lo anterior permitió establecer las mejores relaciones nutricionales para asociarla con la capacidad metabólica del microorganismo en estudio (Tabla 2). 


\section{Respuestas}

Año 17

No. 2

Diciembre 2012 ISSN 0122-820X
Tabla 2. Concentración de carbono y nitrógeno total en las fuentes de crecimiento

\begin{tabular}{|lccc|}
\hline Fuente de crecimiento & \% Carbono & \% Nitrógeno & C/N \\
\hline Sacarosa- $\mathrm{C}_{1}$ & 42,1 & $* \mathrm{ND}$ & 42,1 \\
Melaza-C & & 0,16 & 421,9 \\
Glutamato- $\mathrm{C}_{3}$ & 67,5 & 8,27 & 4,28 \\
Glicerol- $\mathrm{C}_{4}$ & 35,4 & $* \mathrm{ND}$ & 52,9 \\
Soya- $\mathrm{N}_{1}$ & 52,9 & 5,88 & 12,3 \\
Extracto de levadura- $\mathrm{N}_{2}$ & 84,8 & 7,52 & 11,28 \\
\hline
\end{tabular}

*ND: No detectable

\section{Fase screening-Diseño Plackett-Burman}

Los datos para cada uno de los factores analizados no presentaron efectos estadísticamente significativos sobre la variable respuesta $(\mathrm{p}<0,05)$ (Tabla 3).

Tabla 3. Análisis de varianza (Anova) de los resultados obtenidos para determinar los valores de estadístico P, con un nivel de confianza del 95\% del diseño experimental Plackett-Burman.

\begin{tabular}{|cccccc|}
\hline$*$ Símbolo & GL & Estimación & Suma de cuadrados & F-Ratio & P-valor \\
\hline $\mathbf{I}$ & 1 & 0,118333 & 0,126025 & 3,57 & $\mathbf{0 , 0 7 2 1} *$ \\
$\mathbf{N}_{2}$ & 1 & $-0,0872222$ & 0,0684694 & 1,94 & $\mathbf{0 , 1 7 7 7} *$ \\
$\mathbf{C}_{\mathbf{1}}$ & 1 & 0,0794444 & 0,0568028 & 1,61 & $\mathbf{0 , 2 1 7 9} *$ \\
$\mathrm{C}_{2}$ & 1 & $-0,0783333$ & 0,055225 & 1,56 & 0,2242 \\
$\mathrm{C}_{4}$ & 1 & $-0,0783333$ & 0,055225 & 1,56 & 0,2242 \\
$\mathrm{~N}_{1}$ & 1 & $-0,0716667$ & 0,046225 & 1,31 & 0,2649 \\
$\mathrm{~J}$ & 1 & 0,0661111 & 0,0393361 & 1,11 & 0,3027 \\
$\mathrm{~S}_{1}$ & 1 & $-0,0694444$ & 0,0434028 & 1,23 & 0,2796 \\
$\mathrm{~S}_{2}$ & 1 & 0,0105556 & 0,00100278 & 0,03 & 0,8677 \\
$\mathrm{C}_{3}$ & 1 & 0,0283333 & 0,007225 & 0,20 & 0,6555 \\
$\mathrm{~K}$ & 1 & 0,0505556 & 0,0230028 & 0,65 & 0,4282 \\
\hline
\end{tabular}

${ }^{1} \mathrm{GL}$ : Grados de libertad, *Factores significativos sobre el crecimiento del microorganismo. ${ }^{*}$ Codificación de los símbolos nombrados previamente en la tabla 1 .

Con los resultados obtenidos se seleccionaron los factores: aireación (I), hidrolizado de extracto de levadura $\left(\mathrm{N}_{2}\right)$ y la sacarosa $\left(\mathrm{C}_{1}\right)$ que presentaron una significancia representativa, mientras que se descartaron los factores: $\operatorname{MgSO}_{4}\left(\mathrm{~S}_{2}\right)$, glutamato $\left(\mathrm{C}_{3}\right)$ y la solución de microelementos $(\mathrm{K})$ por presentar valores de $\mathrm{P}$ mayores.
Al emplear el análisis matemático se reemplazaron los coeficientes de regresión para cada factor en la ecuación 1 y se estableció el modelo de ajuste de regresión para la productividad en células (Y).

La ecuación lineal del modelo ajustado es:

$$
\mathrm{Y}=9,14083-0,0347222 \cdot \mathrm{S}_{1}+0,00527778 \cdot \mathrm{S}_{2}
$$
$+0,0397222 \cdot \mathbf{C}_{1}-0,0391667 \cdot \mathbf{C}_{2}+0,0141667 \cdot \mathbf{C}_{3}$ $-0,0391667 \cdot \mathrm{C}_{4}-0,0358333 \cdot \mathrm{N}_{1}-0,0436111 \cdot \mathrm{N}_{2}+$ $0,0591667 \cdot \mathbf{I}+0,0330556 \cdot \mathbf{J}+0,0252778 \cdot \mathbf{K}$

Donde $\mathrm{Y}$ es la variable de respuesta denotada como viabilidad celular, $\mathrm{S}_{1}$ y $\mathrm{S}_{2}$ son $\mathrm{K}_{2} \mathrm{HPO}_{4}$ y $\mathrm{MgSO}_{4}$; $\mathrm{C}_{1}, \mathrm{C}_{2}, \mathrm{C}_{3}$ y $\mathrm{C}_{4}$ son fuentes de carbono respectivamente, $\mathrm{N}_{1}$ y $\mathrm{N}_{2}$ son fuentes de nitrógeno, I es aireación, J es agitación y $\mathrm{K}$ es microelementos, permitiendo inferir que la variable más significativa fue la aireación (I) y la variable con el menor efecto fue el $\mathrm{MgSO}_{4}\left(\mathrm{~S}_{2}\right)$.

El suministro de aireación en bajas cantidades (I) favoreció la multiplicación de la bacteria. Este resultado concuerda con lo reportado por autores como Baraket al., (1982); Hartmann y Baldani (2006) donde señalan que a condiciones de baja concentración de oxígeno (microaerofilia) las células de Azospirillum sp. crecen y se multiplican satisfactoriamente, permitiéndoles expresar el mecanismo enzimático de la nitrogenasa (Moure et al; 2012)

Así mismo, el hidrolizado de extracto de levadura $\left(\mathrm{N}_{2}\right)$, mostró el segundo valor más representativo sobre la variable respuesta en un nivel bajo; lo cual demuestra la importancia de este factor en bajas cantidades sobre el crecimiento de A. brasilense C16. Se puede inferir que el anterior resultado pudo estar relacionado con el aporte de elementos nutricionales nitrogenados, azúcares, fosfatos, potasio y diferentes vitaminas del complejo B presentes en esta fuente de crecimiento (Dahm et al., 1993; Rodelas et al., 1993; Bashan et al; 2011)

La sacarosa $\left(\mathrm{C}_{1}\right)$, presentó una influencia 
sobre el crecimiento de la bacteria con una magnitud positiva, por lo que se permite inferir que la relación de las cantidades utilizadas de nitrógeno y carbono en niveles bajos y altos en el diseño respectivamente, contribuye al óptimo desarrollo del microorganismo si se controla adecuadamente de manera que la limitación o el exceso de estos factores nutricionales pueden generar una respuesta desfavorable sobre la capacidad de fijación de nitrógeno (Martínez et al., 1984; Parra y Cuevas, 2001).

Por otra parte, el glutamato $\left(\mathrm{C}_{3}\right)$ fue descartado por ser el segundo factor estadísticamente menos influyente sobre el crecimiento del microorganismo, esto quizás se debió a que este sustrato no pudo ser metabolizado eficientemente por A. brasilense por presentar un esqueleto carbonado - $\alpha$-cetoglutarato- que para esta especie según reportes citados es incapaz de degradarlo. Aunque, también puede atribuirse a que en el medio líquido se encontraban fuentes carbonadas más rápidamente degradables por las diferentes enzimas de las rutas EntnerDoudoroff y Embden-Meyerhof-Parnas (Baldani et al.,1979; Bashan et al., 2011).

A partir de los análisis estadísticos del diseño Plackett-Burman se seleccionaron los tres factores más influyentes (aireación, hidrolizado de extracto de levadura y sacarosa) para ser evaluados en la segunda etapa de optimización, junto con glicerol, hidrolizado de soya y $\mathrm{K}_{2} \mathrm{HPO}_{4}$ para mantener la diversidad nutricional en el medio alternativo.

\section{Fase de optimización-Diseño Box-Behnken}

En esta etapa se evaluaron todas las combinaciones posibles para los tres factores seleccionados $(\mathrm{A}=$ aireación $(\mathrm{I}), \mathbf{B}=$ hidrolizado de extracto de levadura $\left(\mathrm{N}_{2}\right)$ y $\mathrm{C}$ $=$ sacarosa $\left(\mathrm{C}_{1}\right)$ ) mediante la generación de superficies de respuesta (Tabla 4).

Se realizó un análisis de varianza para una regresión múltiple. En general el grado de ajuste del modelo fue del $60,10 \%$ de la variabilidad presentada en la experimentación (Tabla 5)

Tabla 4. Factores influyentes analizados en la etapa de optimización para A. brasilense C16.

\begin{tabular}{|lcccc|}
\hline FACTOR & SíMBOLO & $\begin{array}{c}\text { NIVEL BAJO } \\
(-\mathbf{1})\end{array}$ & $\begin{array}{c}\text { NIVEL MEDIO } \\
\text { (0) }\end{array}$ & $\begin{array}{c}\text { NIVEL ALTO } \\
(+\mathbf{1})\end{array}$ \\
\hline Aireación (I) & A & $1 / 5$ & $2 / 5$ & $3 / 5$ \\
$\begin{array}{l}\text { Hidrolizado de } \\
\text { extracto de } \\
\text { levadura }\left(\mathrm{N}_{2}\right)\end{array}$ & B & $0,424 \mathrm{~g} / \mathrm{L}$ & $0,848 \mathrm{~g} / \mathrm{L}$ & $1,272 \mathrm{~g} / \mathrm{L}$ \\
Sacarosa $\left(\mathrm{C}_{1}\right)$ & $\mathbf{C}$ & $1,5 \mathrm{~g} / \mathrm{L}$ & $3 \mathrm{~g} / \mathrm{L}$ & $4,5 \mathrm{~g} / \mathrm{L}$ \\
\hline
\end{tabular}

Tabla 5. Análisis de varianza (Anova) aplicando el diseño Box-Behnken para A. brasilense C16. Codificación de los factores: $\mathrm{A}=$ aireación (I), $\mathrm{B}=$ hidrolizado de extracto levadura $\left(\mathrm{N}_{2}\right)$ y $\mathrm{C}=$ sacarosa $\left(\mathrm{C}_{1}\right)$.

\begin{tabular}{|lcccc|}
\hline Factor & Suma de cuadrados & F-ratio & Estimación & P-valor \\
\hline $\mathrm{A}=\mathrm{I}$ & 0,0045125 & 0,38 & 0,0475 & 0,5654 \\
$\mathrm{~B}=\mathrm{N}_{2}$ & 0,0153125 & 1,28 & 0,0875 & 0,3086 \\
$\mathrm{C}=\mathrm{C}_{1}$ & 0,0002 & 0,02 & 0,01 & 0,902 \\
$\mathrm{AA}$ & 0,0136641 & 1,15 & 0,121667 & 0,3334 \\
$\mathrm{AB}$ & 0,0009 & 0,08 & 0,03 & 0,7945 \\
$\mathrm{AC}$ & 0,013225 & 1,11 & 0,115 & 0,3406 \\
$\mathrm{BB}$ & 0,0152026 & 1,27 & $-0,128333$ & 0,3102 \\
$\mathrm{BC}$ & 0,015625 & 1,31 & 0,125 & 0,3042 \\
$\mathrm{CC}$ & 0,00862564 & 0,72 & 0,0966667 & 0,4339 \\
\hline
\end{tabular}

Se pudo observar que la interacción entre los factores hidrolizado de extracto de levadura (B) y sacarosa (C) presentó un efecto positivo sobre la variable de respuesta con una mayor significancia que los demás factores, de modo que existe influencia conjunta sobre el crecimiento de Azospirillum brasilense C16. Simultáneamente, se examinó el efecto independiente de los factores dando como resultado que el factor sacarosa $(\mathrm{C})$, tiene el menor efecto individual por lo que debe existir una relación proporcional entre $\mathrm{C} / \mathrm{N}$ en el medio de cultivo para que esta fuente (C) tenga un efecto óptimo sobre la variable de respuesta. ISSN 0122-820X 
aireación (A) no se observa claramente una tendencia hacia los dos niveles evaluados $(-1$ y 1) debido a que se presenta similitud en la concentración del microorganismo; mientras que se observa una menor curvatura en los puntos centrales por lo que nos permite inferir una baja tasa de multiplicación de la bacteria en estas magnitudes. Para el hidrolizado de extracto de levadura (B), las máximas productividades se obtuvieron cuando esta variable se encontró en el nivel 1 manteniendo constante la sacarosa (C) (Figura 1A).

Figura 1. Superficies de respuesta de la interacción entre el factor aireación (A), hidrolizado de extracto de levadura (B) y la sacarosa (C).
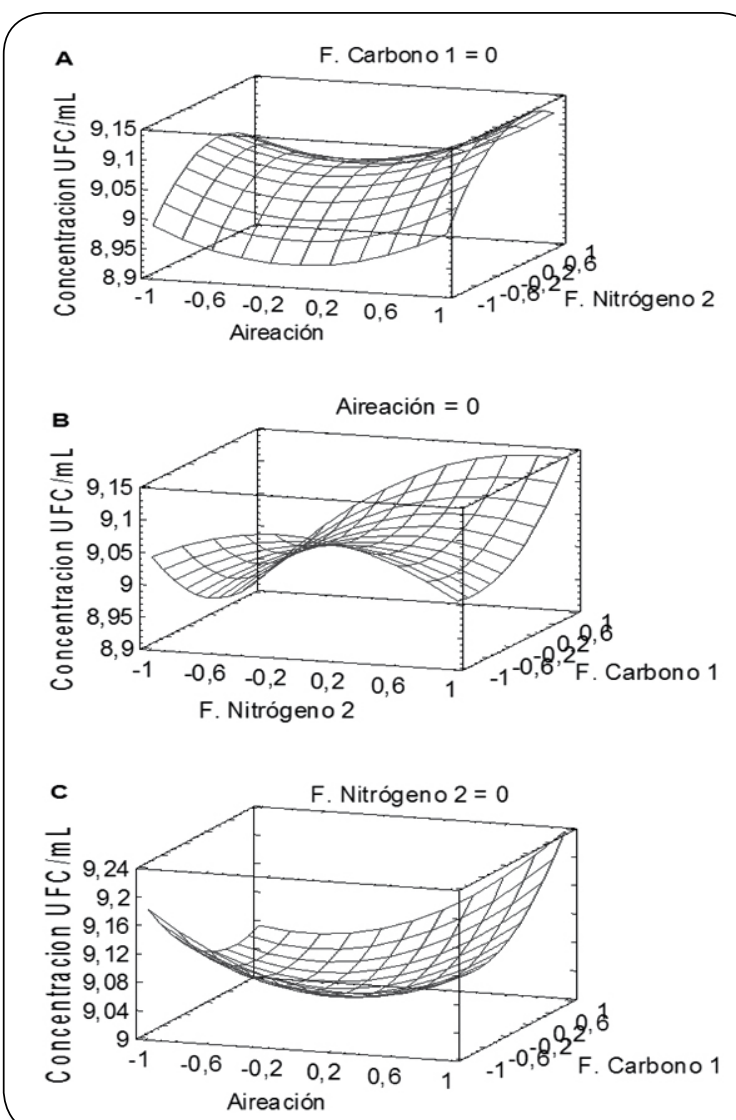

Cuando la magnitud de aireación (A) se mantuvo constante, se observa que el factor hidrolizado de extracto de levadura (B) presentó una tendencia hacia los puntos centrales con una mayor curvatura en la gráfica, lo que confirma que la formulación de esta cantidad en el nivel medio es la más apropiada para el crecimiento de Azospirillum brasilense C16. La respuesta del factor sacarosa (C), reflejó un máximo crecimiento del microorganismo en la región del espacio explorada cuando este factor está en un nivel 1 (Figura 1B).

Cuando el hidrolizado de extracto de levadura (B) se mantuvo constante, se observa el efecto de los niveles evaluados para el factor aireación (A) sobre la variable respuesta. A su vez, la sacarosa (C), mostró una tendencia a maximizar la respuesta si se utiliza un nivel de 1 (Figura 1C).

Mediante el análisis estadístico se obtuvo los coeficientes de regresión generados, reemplazándolos en la ecuación 1 del modelo de regresión se obtiene:

$$
\begin{aligned}
& \mathrm{Y}=9,04333+0,02375 \cdot \mathrm{A}+0,04375 \cdot \mathrm{B} \\
& +0,005 \cdot \mathrm{C}+0,0608333 \cdot \mathrm{A}^{2}+0,015 \cdot \mathrm{AB}+ \\
& 0,0575 \cdot \mathrm{AC}-0,0641667 \cdot \mathrm{B}^{2}+0,0625 \cdot \mathrm{BC}+ \\
& 0,0483333 \cdot \mathrm{C}^{2}
\end{aligned}
$$

La composición del medio de cultivo estandarizado fue de: $3 \mathrm{~g} / \mathrm{L}$ sacarosa $\left(\mathrm{C}_{1}\right), 6,75$ $\mathrm{mL} / \mathrm{L}$ melaza $\left(\mathrm{C}_{2}\right), 0,77 \mathrm{~mL} / \mathrm{L}$ glicerol $(\mathrm{C} 4)$, $14,42 \mathrm{~mL} / \mathrm{L}$ hidrolizado de soya $\left(\mathrm{N}_{1}\right), 0,848$ $\mathrm{g} / \mathrm{L}$ hidrolizado de extracto de levadura $\left(\mathrm{N}_{2}\right)$, 0,25 g/L K $\mathrm{HPO}_{4}(\mathrm{~S} 1), 3 / 5$ aireación y una agitación de $120 \mathrm{rpm}$.

\section{Cinética de crecimiento en el medio de cultivo tradicional y alternativo}

Se determinó la cinética de crecimiento de Azospirillum brasilense C16 tanto en el medio de control y alternativo. Se obtuvo una concentración de 1 X $10^{9} \mathrm{UFC} / \mathrm{mL}$ en un tiempo óptimo de fermentación de 26 horas en el medio alternativo; mientras que en el medio control se obtuvo la misma concentración en menor tiempo. Sin embargo, el medio de cultivo control se consumió mucho más rápido descendiendo drásticamente su concentración (Figura 2).

En contraste, se linealizó la curva de crecimiento de A. brasilense C16 en los medios evaluados, obteniendo los parámetros 
Estandarización de un medio de cultivo a partir de fuentes agroindustriales para la multiplicación de

cinéticos: velocidad específica de crecimiento $(\mu \mathrm{x})$ y el tiempo de duplicación ( $t d)$ (Tabla 6).

Figura 2. Cinética de crecimiento para A. brasilense C16 en función del tiempo en el medio control-Dygs y medio de cultivo alternativo.

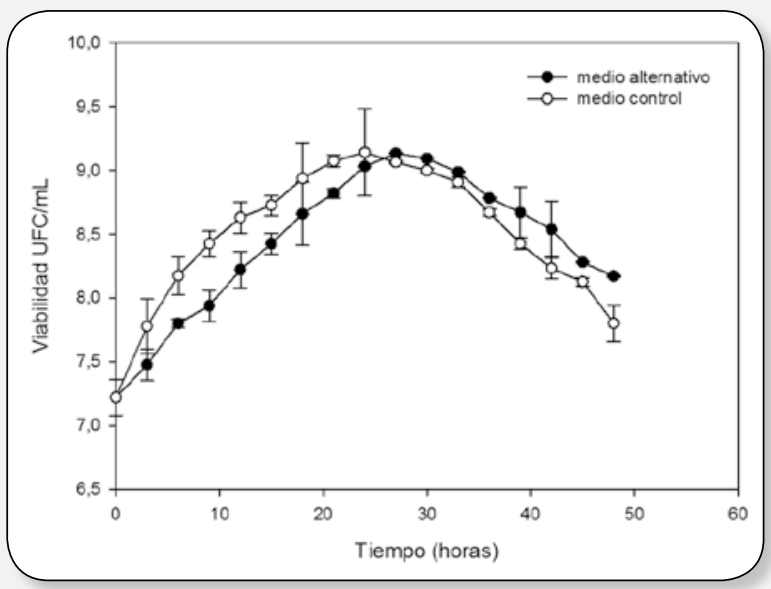

Tabla 6. Parámetros cinéticos para A. brasilense C16 en el medio control y medio alternativo.

\begin{tabular}{|ccc|}
\hline Parámetro Cinéticos & Medio control & Medio alternativo \\
\hline $\begin{array}{c}\text { Velocidad específica de } \\
\text { crecimiento }(\mu x)\end{array}$ & $0,25 \mathrm{~h}^{-1}$ & $0,22 \mathrm{~h}^{-1}$ \\
Tiempo de duplicación $(t d)$ & $2,77 \mathrm{~h}$ & $3,15 \mathrm{~h}$ \\
\hline
\end{tabular}

Al analizar los resultados obtenidos, se puede discutir que las fuentes de crecimiento complejas utilizadas en el medio alternativo requieren de un gran número de reacciones metabólicas para degradar estos sustratos por el microorganismo; asegurando de esta manera la multiplicación efectiva de la bacteria por más tiempo en comparación con los sustratos utilizados en el medio control; los cuales son degradados más rápidamente ocasionando el consumo de estos en menor tiempo.

El análisis costo-beneficio realizado a la tecnología generó una reducción de los costos, al reemplazar insumos grado reactivo en el medio control por fuentes nutricionales alternativas, por un valor de $\$ 85,43$ por litro en el medio alternativo; mientras que el costo del medio de referencia tiene un precio estimado de \$2922,76.
Es importante resaltar que aunque la concentración fue similar en ambos medios de cultivos durante el tiempo evaluado, se destaca el menor costo con el medio estandarizado utilizando sustratos agroindustriales.

\section{Conclusiones}

La aplicación de herramientas estadísticas y de diseños de experimentos demostraron ser estrategias útiles y eficientes para llevar a cabo la optimización de medios de cultivo. El análisis demostró que la producción de biomasa en A. brasilense C16 esta determinada por la aireación, hidrolizado de extracto de levadura y la sacarosa.

El diseño de Box-Behnken demostró que para mantener una adecuada concentración de Azospirillum brasilense C16, es preciso que el balance de las magnitudes de los factores sean cuidadosamente controladas manteniendo estables la relación de aireación y agitación. El medio de cultivo alternativo para Azospirillum brasilense C16 mostró una economía del 95\% en comparación con el medio de referencia.

\section{Referencias Bibliográficas}

ADESEMOYE, A.O., TORBERT, H.A., KLOEPPER, J.W. 2008. Enhanced plant nutrient use efficiency with PGPR and AMF in an integrated nutrient management system. Can. J.Microbiol.54: 876-886.

BALDANI, V.L.D., FERREIRA, J., TEIXEIRA, K., BALDANI, J.I., REIS, V. 2008. Cap. Inoculants based on nitrogen fixing bacteria Azospirillum spp and their application in tropical agriculture. ISBN: 978987-98475-8-9 225-232. ISSN 0122-820X 
BASHAN, Y., TREJO, A., DE-BASHAN, L.E. 2011. Development of two culture media for mass cultivation of Azospirillum spp. and for production of inoculants to enhance plant growth. Biol Fertil Soils 47:963-969.

BOX G, BEHNKEN D. 1960. Some new three level designs for the study of quantitative variables Technometrics. 2(4): 455-475.

CÁRDENAS, D.M., GARRIDO, M., BONILLA, R., BALDANI, V.L. 2010. Aislamiento y caracterización de cepas de Azospirillum sp. en pasto guinea (Panicum maximum jacq.) del valle del Cesar. Pastos y Forrajes 33(3):1-10.

DAHM,H., ROZYCKI,H.,STRZELCZYK, E., L.I C.Y. 1993. Production of B-group vitamins by Azospirillum spp. grown in media of different $\mathrm{pH}$ at different temperatures. $\mathrm{Z}$. Mikrobiol. 148: p. 195-203.

DÍAZ-ZORITA, M., FERNÁNDEZCANIGIA, M.V. 2009. Field performance of a liquid formulation of Azospirillum brasilense on dryland wheat productivity. European journal of soil biology. 45: 3-11.

DOBBELAERE, S., VANDERLEYDEN, J., OKON Y. 2003. Plant growth promoting effects of diazotrophs in the rhizosphere, Crit. Rev. Plant Sci. 22: 107-149.

DÖBEREINER, J, BALDANI, V.L.D., BALDANI, J.I. 1995. Como isolar e identificar bactérias diazotróficas de plantas ñao leguminosas. Embrapa-CNPAB.

DOYLE, M., BEUCHAT, L., MONTVILLE, T. 2000. Microbiología de los alimentos fundamentos y fronteras. España: Acribia. p. 312-320

GAMARRA, J.R. 2005. La economía del Cesar después del algodón. En: Documentos de trabajo sobre economía regional. No. 59. $116 \mathrm{p}$.

HARTMANN, A. BALDANI, J.I. 2006. Chapter 3.1.5. The Genus Azospirillum. Prokaryotes 5: 115-140. DOI: 10.1007/0387-30745-1_6.
MARTINEZ-DRETS, G, DEL GALLO, M., BURPEE C., BURRIS, R.H. 1984. Catabolism of carbohydrates and organics acids and expression of nitrogenase by Azospirilla, 1984. J. Bacteriol. 159: 80-85.

MOURE, V.R., DANYAL, K., YANG, Z.Y., WENDROTH, S., MÜLLER-SANTOS, M., PEDROSA, F.O., SCARDUELLI, M., GERHARDT, E.C., HUERGO, L.F., SOUZA, E.M., SEEFELDT, L.C. 2012. The Nitrogenase Regulatory Enzyme Dinitrogenase Reductase ADP-ribosyltransferase (DraT) is Activated by Direct Interaction with the Signal Transduction Protein GlnB. J. Bacteriol. doi:10.1128/JB.01517-12. p. 1-28.

NILADEVI, K.N., SUKUMARAN, R.K., JACOB, N., ANISHA, G.S., PREMA, P. 2009. Optimization of laccase production from a novel strain-Streptomyces psammoticus using response surface methodology Microbiological Research 164: 105-113.

PARRA, Y., CUEVAS, F. 2001. Potencialidades de Azospirillum como inoculante para la agricultura. Cultivos Tropicales, 23: p. 31-41.

PLACKETT, R., BURMAN, J. 1946. The design of optimum multifactorial experiments. Biometrika 33(4): 305-325.

RODRIGUES, J., MALAVOLTA, V.A., VICTOR. O. 1986. Meio simples para o isolamento e cultivo de Xanthomonas campestris pv. citri tipo B. Summa Phytopathologica, Campinas, v. 12, n. 1-2, p. 16.

RODELAS, B.V., SALMERON, V., MARTINEZ-TOLEDO and GONZALEZLOPEZ, M. 1993. Production of vitamins by Azospirillum brasilense in chemically-defined media. Plant. Soil. 153: p. 97-10.1 